\title{
Gerenciamento de semáforos e análise do impacto da dispersão de poluentes utilizando o simulador SUMO
}

Traffic lights management and analysis of the impact of the dispersion of pollutants using the simulator SUMO

\author{
M. B. Born ${ }^{1 *}$; D. F. Adamatti ${ }^{1}$; M. S. Aguiar $^{2}$ \\ ${ }^{1}$ Centro de Ciências Computacionais - C3/Lamsa, Universidade Federal do Rio Grande - FURG, 96203-900, Rio \\ Grande-RS, Brasil \\ ${ }^{2}$ Programa de Pós-Graduação em Computação, Universidade Federal de Pelotas - UFPel, 96010-610, Pelotas-RS, \\ Brasil
}

*miriamborn@gmail.com

(Recebido em 22 de outubro de 2014; aceito em 07 de março de 2015)

$\mathrm{Na}$ atualidade, a mobilidade urbana e a qualidade do ar são assuntos de destaque, devido ao tráfego intenso de veículos e a emissão de poluentes dissipada na atmosfera. Na literatura, foi proposto um modelo de controle otimizado de semáforo utilizando Algoritmos Genéticos (AG). Estes algoritmos foram introduzidos neste contexto de controle de trânsito, buscando possíveis soluções para a problemática de semáforos nos grandes centros urbanos. A análise da dispersão de poluentes torna-se um aspecto importante de ser estudado e avaliado, para que soluções viáveis sejam implantadas visando a melhoria do tráfego urbano e da qualidade do ar. Assim, este trabalho tem como objetivo o desenvolvimento de um Algoritmo Genético (AG) capaz de auxiliar no gerenciamento de semáforos do centro da cidade de Rio Grande/RS e analisar, a partir de cenários simulados no simulador SUMO (Simulation of Urban Mobility), o impacto da dispersão de poluentes nesta área.

Palavras-chave: Algoritmos Genéticos, Dispersão de Poluentes e SUMO.

\begin{abstract}
Nowadays, urban mobility and air quality issues are prominent, due to the heavy traffic of vehicles and the emission of pollutants dissipated in the atmosphere. In the literature, a model of optimal control of traffic lights using Genetic Algorithms (GA) has been proposed. These algorithms have been introduced in the context of traffic control, in order to search for possible solutions to the problems of traffic lights in major urban centers. The analysis of pollutant dispersion becomes an important aspect to be studied and evaluated, so viable solutions are deployed in order to improve the urban traffic and air quality. Thus, this work aims to develop a Genetic Algorithm (GA) can help manage traffic lights from the city of Rio Grande / RS center and analyze, from simulated scenarios in the simulator SUMO (Simulation of Urban Mobility) the impact of the dispersion of pollutants in this area.
\end{abstract}

Keywords: Genetic Algorithms, Dispersion of Pollutants and SUMO.

\section{INTRODUÇÃO}

Anualmente, a frota de veículos nos grandes centros urbanos cresce a cada ano, causando problemas de tráfego para motoristas, pedestres e para o meio ambiente [1]. A travessia de pedestres nas faixas de cruzamento de semáforos apresentam riscos significativos em diversos países, como China e Espanha, devido a este crescimento extremo. Segundo [2], a cada ano, aproximadamente 500 mil pedestres são mortos nos cruzamentos destes países. Os controladores de semáforos convencionais realizam a troca de estágios de forma constante, sendo que o sistema calcula o tempo de ciclo com base na carga média de tráfego, desconsiderando a dinâmica natural do mesmo, agravando problemas de congestionamento e contribuindo para a dispersão de poluentes na atmosfera.

$\mathrm{Na}$ literatura, vários trabalhos aplicam técnicas da Inteligência Artificial (IA), tais como Sistemas Multiagentes (SMA) e Computação Evolucionária (CE), no contexto de gerenciamento de recursos naturais devido à forte influência desta área na qualidade de vida da população. A dispersão de poluentes é objeto de estudo, principalmente nos médios e grandes 
centros urbanos e industriais, como a cidade de Rio Grande/RS, onde existem fontes emissoras de poluentes e o tráfego de veículos é intenso [3].

No trabalho de [4] pode-se constatar a aplicação da Inteligência Artificial (IA) no controle do tráfego rodoviário com o objetivo de otimizar os tempos de semáforos. Em [5] foi proposto o controle de semáforos utilizando Redes Neurais Artificiais com Funções de Base Radial com o propósito de melhorar o desempenho do tráfego. Segundo [6] o emprego de Sistemas Multiagentes (SMA) no controle de tráfego urbano torna-se bastante viável, visando analisar o grau de impacto das escolhas dos motoristas no sistema de tráfego.

Em [7] apresentam-se medidas que favorecem a mobilidade e acessibilidade de pedestres nas áreas urbanas, identificando assim, problemas referentes à acidentes com pedestres. E, outro trabalho relatado neste artigo, que menciona o estudo de recursos naturais e dispersão de poluentes foi proposto por [8], onde foi realizado o estudo da dispersão de poluentes atmosféricos através da aplicação de modelos computacionais, considerando a cidade de Rio Grande/RS como estudo de caso.

Desta forma, o monitoramento e controle de tráfego e da dispersão de poluentes torna-se um desafio para as autoridades de transporte no mundo todo [2].

Neste contexto, pretende-se desenvolver um Algoritmo Genético (AG) que simule primeiramente duas junções de trânsito e, logo após todas as junções do centro de uma cidade que possui tráfego de veículos intenso. Neste caso de estudo será a cidade de Rio Grande/RS. A ferramenta SUMO será utilizada para o desenvolvimento destas simulações, bem como a análise da dispersão de poluentes nesta área específica.

Este artigo apresenta os resultados parciais obtidos no estágio atual de desenvolvimento do trabalho, onde um Autômato Celular (AC) para duas junções de trânsito e o Diagrama de Classes foi elaborado com o intuito de compreender a dinâmica de controle dos semáforos. Também apresentam-se as configurações dos mapas de ruas e semáforos do simulador SUMO e o estudo da dispersão de poluentes nessa ferramenta. No presente momento está sendo desenvolvido o Algoritmo Genético a ser utilizado nas simulações e análises.

Este artigo está estruturado da seguinte forma: na Seção 2 são descritos os materiais e métodos utilizados neste artigo e nas respectivas subseções são apresentados estudos sobre Algoritmos Genéticos (AG), a ferramenta SUMO e a Dispersão de Poluentes e por fim, a seção proposta para gerenciamento de semáforos e análise de dispersão de poluentes. Na Seção 3 são apresentados os resultados preliminaries desta proposta e na Seção 4 são apresentadas as conclusões preliminares e os trabalhos futuros.

\section{MATERIAL E MÉTODOS}

A proposta do presente trabalho é desenvolver um Algoritmo Genético (AG), que simule as junções do centro da cidade de Rio Grande/RS, onde o tráfego de veículos é intenso, visto que a população do município teve um crescimento elevado devido à concentração de empresas do polo naval.

As Subseções a seguir descrevem o estudo dos assuntos pertinentes à realização deste trabalho. Na Seção 2.1 são descritas características dos Algoritmos Genéticos (AG), destacando assim a escolha destes no desenvolvimento no trabalho. A Seção 2.2 descreve o software SUMO, bem como a biblioteca de dispersão de poluentes que a ferramenta possui e por fim, na Seção 2.3 a proposta de gerenciamento de semáforos e a análise da dispersão de poluentes que será realizada neste trabalho.

\subsection{Algoritmos Genéticos}

Os Algoritmos Genéticos (AG) são técnicas de busca baseadas Teoria da Evolução introduzida por Charles Darwin (1809-1882). Nos AG's as variáveis de determinado problema são representadas como genes em um cromossomo que também pode ser denominado indivíduo [9]. 
O sistema proposto por Holland era composto por uma cadeia de bits (0's e 1's), chamados indivíduos. Estes indivíduos evoluíam até encontrar um melhor cromossomo que atendesse um problema específico. A solução do sistema era encontrada de modo automático e nãosupervisionado, sendo que as informações fornecidas a este compreendiam os ajustes de cada cromossomo.

Para o funcionamento dos AG's é necessário, antes de sua execução, atribuir uma codificação de acordo com o problema em questão e definir uma função de fitness, também chamada grau de adaptação. Esta função deve retornar um valor numérico proporcional a utilidade ou habilidade do indivíduo que o cromossomo representa.

No decorrer da execução, alguns pais são selecionados para a reprodução e posteriormente recombinados para gerar os filhos. A partir do problema definido os AG's dividem-se nas seguintes etapas: inicialização, avaliação, seleção, reprodução, eliminação, teste de satisfação e solução. Sendo importante observar que a função de avaliação (fitness) dependerá do problema em questão [10]11].

\subsection{SUMO e Dispersão de Poluentes}

O SUMO (Simulation of Urban MObility) é um simulador microscópico de trânsito, que foi desenvolvido em 2001 pelo Centro Aeroespacial Alemão (DLR), com o intuito de auxiliar a comunidade de pesquisa de tráfego com uma ferramenta onde algoritmos pudessem ser implementados e avaliados, sem a necessidade de obter um tráfego completo de simulação. Possui código aberto, portátil e projetado para simular modelagens de redes rodoviárias de grande porte [12].

Dentre as principais características de SUMO destacam-se: possui todas as aplicações necessárias para simular uma rede de tráfego, diferentes tipos de veículos, os movimentos dos veículos ocorrem em espaço contínuo e tempo discreto, ruas com múltiplas faixas e mudança de faixa, interface gráfica para os usuários, velocidade de execução nas simulações, interoperabilidade com demais aplicações em tempo de execução, entre outras.

O simulador possui em sua configuração definições tais como: fundo de ambiente, ruas, nodos, veículos, entre outros. A partir das definições de veículos com relação às cores destes, podemos selecionar como definição para a simulação, por exemplo, emissões de $\mathrm{CO} 2, \mathrm{CO}, \mathrm{NOx}$ e outros [12].

Desta forma, os veículos mudam as cores, nas quais pode-se caracterizar como emissão de poluentes para simular uma determinada rede de estradas, no caso deste trabalho uma parte do centro da cidade de Rio Grande e medir qual a emissão de poluentes de determinado número de veículos em um tempo específico.

Por padrão, o SUMO é capaz de simular as emissões de poluentes veiculares com base no banco de dados da HBEFA (Handbook Emission Factors for Road Transport). Este órgão fornece fatores de emissão de poluentes para todas as categorias de veículos. Os poluentes escolhidos para ser implementado neste simulador são: CO2, CO, HC, NOx, PMX e Consumo de combustível [12]. A Figura 1 representa o ambiente do simulador SUMO e os poluentes emitidos pelos veículos da simulação. 


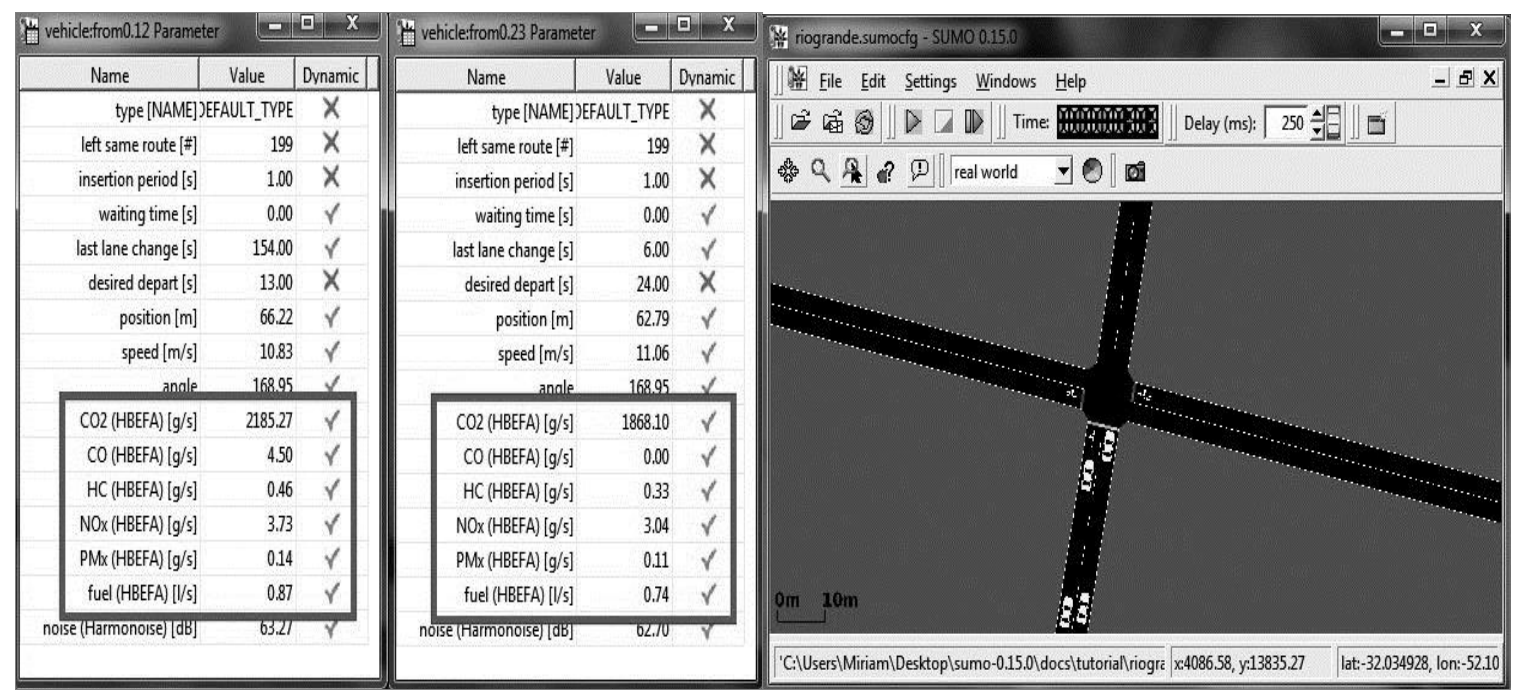

Figura 1. Representação de junção com semáforo e poluentes no SUMO

\subsection{Proposta para Gerenciamento de Semáforos e Análise de Dispersão de Poluentes}

A proposta do presente artigo consiste em estender a aplicação do algoritmo desenvolvido no artigo [2], em um primeiro momento, para duas junções de tráfego e, posteriormente para os demais cruzamentos do centro da cidade de Rio Grande/RS, nos modos estático e dinâmico de um AG.

Para o desenvolvimento deste trabalho utilizou-se como metodologia o modelo do algoritmo de [2], juntamente com o Autômato Celular (AC), com os estados dos possíveis trajetos que os veículos podem percorrer ao longo da execução. A linguagem definida para a implementação foi Java, visto que dentre as suas diversas características, a independência de plataforma e a portabilidade são requisitos interessantes no auxílio do problema em questão.

O propósito do trabalho de [2] foi desenvolver um controle otimizado de semáforo utilizando Algoritmo Genético (AG) em quatro vias, uma junção e uma travessia de pedestre. Os Algoritmos Genéticos foram introduzidos neste contexto de controle de trânsito com intuito de fornecer uma resposta inteligente de intervalo verde baseado em dados de carga de tráfego dinâmico, buscando superar os diversos problemas dos controles convencionais de tráfego de veículos.

No modelo de semáforos proposto, os autores simularam cinco sensores, sendo quatro destes para detecção do número de veículos em cada pista e o quinto sensor detecta o número de pedestres na fila para travessia. O sistema calcula para dois modos, estático e dinâmico, quanto tempo um determinado veículo $\mathrm{V}$ leva para ir de uma origem $\mathrm{X}$ ao destino $\mathrm{Y}$ e para $\mathrm{o}$ desenvolvimento do algoritmo do modelo foram definidas variáveis de entrada e saída.

De acordo com [2] uma das formas de projetar e simular regras de condução simples de veículos é utilizar Autômato Celular (AC), pois estes permitem representar eventos significativos que ocorrem durante congestionamentos, paralisação do tráfego, retorno de movimento, regressar até parar novamente e assim por diante. Os atributos identificados para os veículos do modelo compreendem a velocidade média, máxima e desejada, localização, aceleração atual e $i d$ do veículo.

Posteriormente, os parâmetros para o modelo foram identificados e definidos para veículos e pista, como segue:

- Comprimento da pista: a pista é dividida em um conjunto de 15 células e possui um único $i d$, sendo o tamanho de cada célula 16 pixels e um veículo ocupa duas células, ou seja, 32 pixels. As células de cada pista são representadas por uma matriz, permitindo ao algoritmo verificar se estas estão vazias ou ocupadas e verificar se os veículos estão na posição real; 
- Número de veículos: indica o número de veículos em uma pista;

- Velocidade dos veículos: este parâmetro representa o número de células percorridas por um veículo em determinado momento, sendo que esta pode variar de 0 a 5 células por segundos;

- Os sinais de trânsito: este modelo possui quatro semáforos para o controle de tráfego e um para a travessia de pedestre. Cada semáforo possui um id e os modos de sinal amarelo, vermelho e verde. As quatro vias, junção de duas pistas, tem um nó de entrada, saída e interseção. $\mathrm{O}$ nó de cruzamento consiste de quatro células, cada uma com sua identificação e a área de travessia de pedestres é identificada através das pistas B e B1. Neste ambiente cada veículo pode mover-se para frente, permanecer na posição e virar à esquerda ou à direita.

No modelo do Algoritmo Genético (AG) de [2] foram estabelecidas as etapas: inicializar população, avaliar população, seleção de cromossomos e recombinação de cromossomos. Sendo que, para a etapa de seleção dos cromossomos foi utilizado o método da roleta com probabilidade de seleção proporcional a função de aptidão (fitness), método muito utilizado nestes casos.

Os resultados obtidos no trabalho de [2], comparando os modos estático e dinâmico, concluíram que o modo dinâmico, com a utilização dos AG's, possui melhor desempenho se comparado ao modo estático. Devido à sua flexibilidade, o modelo de controle dinâmico é capaz de calcular o tempo de verde ideal com base no número de veículos e pedestres no sinal vermelho e o comprimento da fila de veículos e pedestres.

Os resultados também mostram que ganho de tempo de um veículo que viaja através do sistema de semáforos controlados por AG’s é significativo.

\section{RESULTADOS}

A partir da metodologia utilizada no trabalho de [2], desenvolveu-se neste artigo um autômato celular com os estados, ou seja, as pistas de origem e destino que os veículos percorrerão, com duas junções, como representado na Figura 2. Nesta representação, q0 corresponde ao estado inicial do autômato e $q 19$ o estado final. Os demais estados são intermediários e as letras $A, B, C, \ldots, G, B 1, C 1, \ldots, G 1$ representam as pistas.

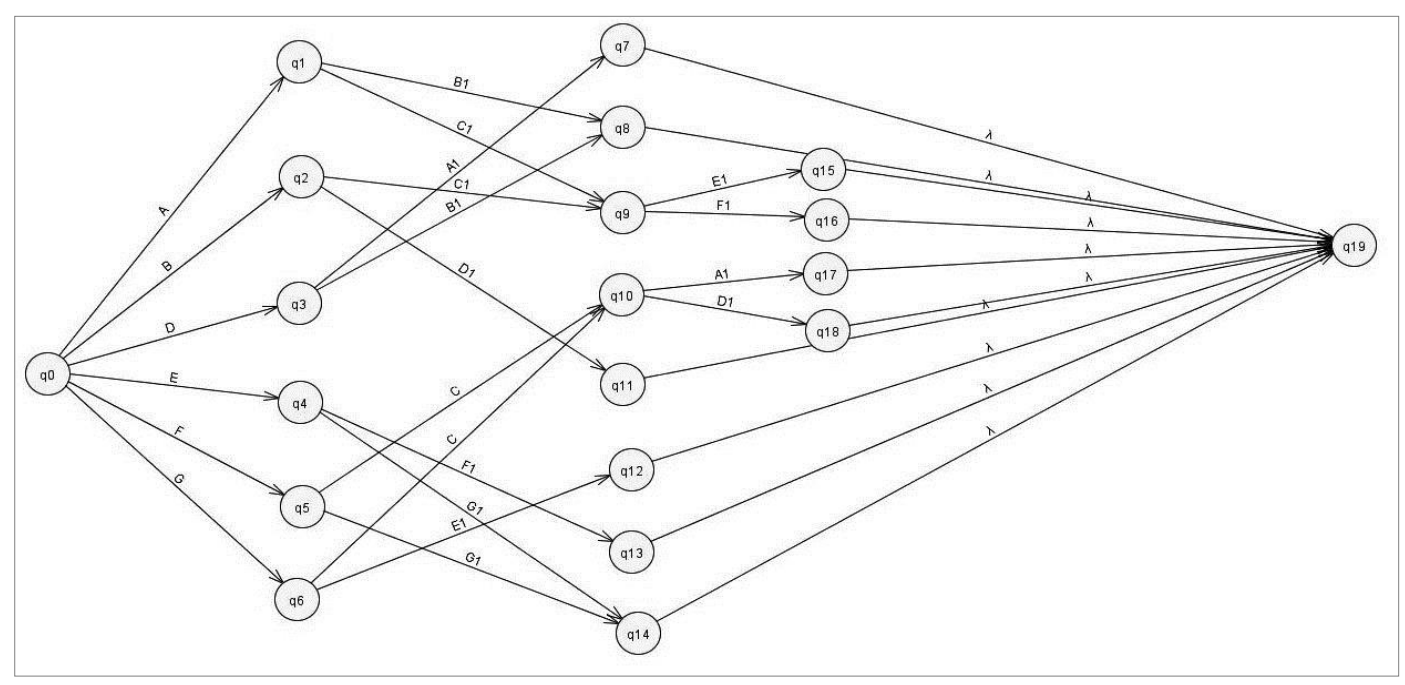

Figura 2. Autômato Celular para modelo de tráfego

Concomitantemente está sendo desenvolvido o diagrama de classes desta proposta. Este, por sua vez, poderá ser alterado conforme o andamento do trabalho. Na Figura 3 são representadas as classes inicialmente identificadas neste trabalho. 
Entretanto, no trabalho proposto será desenvolvido um Algoritmo Genético (AG) para auxiliar no gerenciamento de semáforos visando analisar e avaliar o impacto da dispersão de poluentes a partir de cenários simulados no SUMO, considerando como estudo de caso o centro da cidade de Rio Grande/RS.

Nesta etapa inicial do desenvolvimento do AG será admitido a análise dos tempos de semáforos apenas para os veículos e após a conclusão desta fase, a modelagem do Autômato Celular para a análise dos tempos de semáforos com relação aos pedestres.

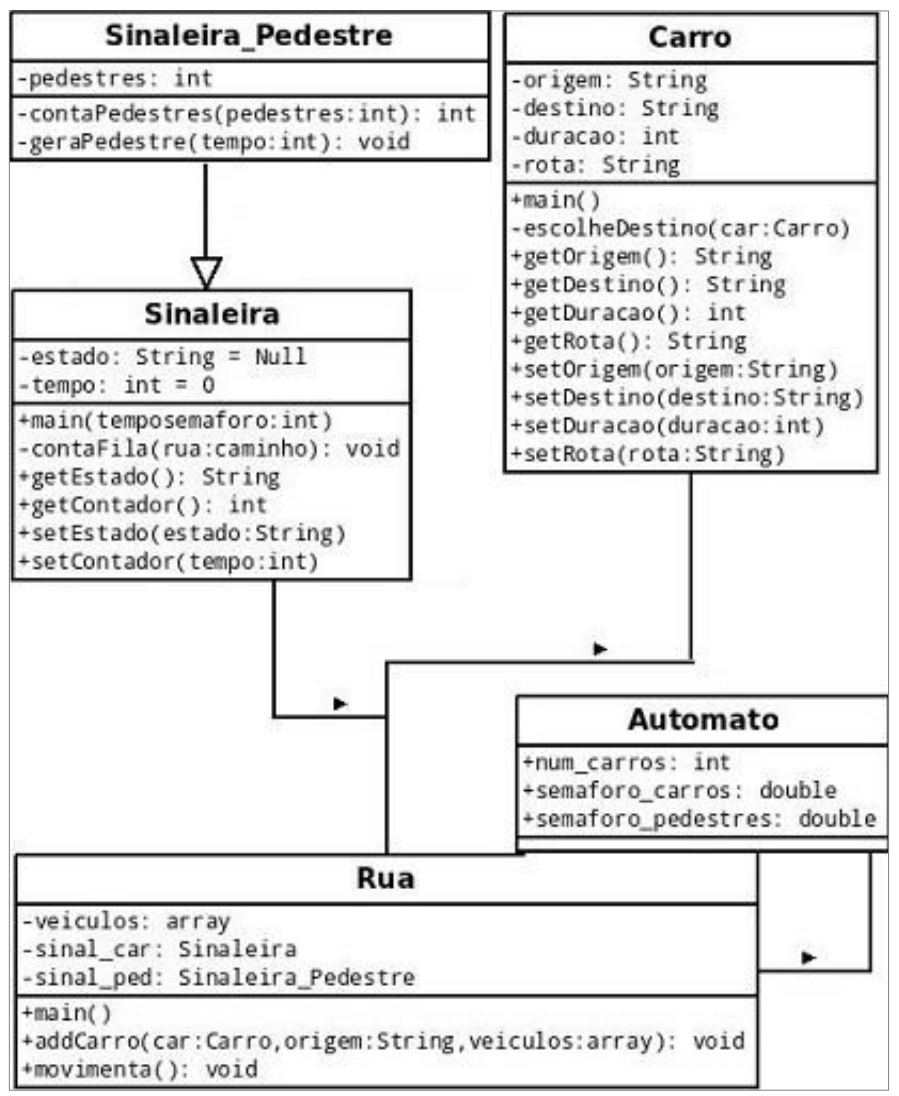

Figura 3. Diagrama de Classes do sistema proposto

Cada uma destas classes possui os atributos e métodos que estão sendo desenvolvidos e as relações entre elas. De forma que a classe Sinaleira_Pedestre se relaciona diretamente com a classe Sinaleira, que por sua vez tem relação com a classe Carro. Por fim, a classe Rua se relacionará com a classe Automato.

O número de estados do Autômato Celular (AC) e as classes do diagrama aumentam consideravelmente à medida que os novos cruzamentos são inseridos no autômato, sendo que esta etapa da proposta encontra-se em andamento.

\section{CONCLUSÕES E TRABALHOS FUTUROS}

O problema de gerenciamento de semáforos e a dispersão de poluentes cresce a cada ano devido ao tráfego intenso de veículos, principalmente nas grandes cidades. Estudos e análises destes assuntos tornam-se necessários para melhorar a mobilidade urbana das cidades e diminuir o impacto da emissão de poluentes na atmosfera.

$\mathrm{O}$ artigo utilizado como referência para este trabalho mostrou a comparação dos resultados das simulações realizadas com o Algoritmo Genético (modo dinâmico) e modo estático, como representado no mesmo pode-se concluir que a utilização de um AG melhora consideravelmente 
o tempo de espera em semáforos, tanto para veículos como para pedestres. Desta forma, buscase para este trabalho esta melhora de tempo de espera para minimizar os efeitos da dispersão de poluentes produzidos pelos veículos, utilizando como estudo de caso a cidade de Rio Grande/RS.

Como trabalhos futuros, pretende-se a conclusão da modelagem proposta, bem como a extensão do Autômato Celular e do Diagrama de Classes para as ruas do centro da cidade de Rio Grande e a aplicação do Algoritmo Genético nas simulações para que o impacto da dispersão de poluentes nesta área seja analisado.

Este trabalho visa contribuir de forma que, pesquisadores e estudantes, possam desenvolver modelos de tráfego aproximando-se do mundo real, ou seja, estender a modelagem proposta à realidade do país ou de cada região, contribuindo para solução de controladores de tráfego e a emissão de poluentes no meio ambiente.

\section{REFERÊNCIAS BIBLIOGRÁFICAS}

1. Born MB, Adamatti DF. Um Experimento sobre Dispersão de Poluentes para a Cidade de Rio Grande/RS utilizando o Simulador SUMO. In: IV Simpósio de Tecnologia da Informação do Noroeste Do RS, Cruz Alta/RS. 2013.

2. Turky M, Ahmad S, Yusoff M. The Use of Genetic Algorithm for Traffic Light and Pedestrian Crossing Control. International Journal of Computer Science and Network Security. 2009 February;9(2):88-96.

3. Nunes G. Estudo e análise da Dispersão de Poluentes: um estudo de caso para a cidade de Rio Grande/RS. Dissertação (Mestrado em Modelagem Computacional) — Universidade Federal do Rio Grande, Rio Grande/RS. 2013.

4. Silva E, Manfredini V. Aplicação de Conceitos da Inteligência Artificial no Controle de Tráfego Rodoviário. In: Trabalho de Conclusão de Curso (Graduação em Ciência da Computação). São Paulo/SP. 2010.

5. Heinen M, Sá C, Silveira F, Cesconetto C, Sohn G. Controle Inteligente de Semáforos Utilizando Redes Neurais Artificiais com Funções de Base Radial. In: Encontro Anual de Tecnologia da Informação e Semana Acadêmica de Tecnologia da Informação. Frederico Westphalen/RS; 2013. p.38-45.

6. Retore P, Santos R, Marietto M, Sá C. Sistemas Multi-Agentes Reativos Modelando o Controle de Tráfego Urbano. Hífen. 2006;30(58):81-87.

7. Melo FB. Proposição de Medidas Favorecedoras à Acessibilidade e Mobilidade de Pedestres em áreas Urbanas. Estudo de Caso: O Centro de Fortaleza. Dissertação (Mestrado em Engenharia de Transportes) - Universidade Federal do Ceará, Fortaleza/CE. 2005.

8. Nunes G. Estudo e análise da Dispersão de Poluentes: um estudo de caso para a cidade de Rio Grande/RS. Dissertação (Mestrado em Modelagem Computacional) - Universidade Federal do Rio Grande, Rio Grande/RS. 2013.

9. Aguiar MS. Análise Formal da Complexidade de Algoritmos Genéticos. Dissertação (Mestrado em Ciência da Computação) - PPGC/UFRGS, Porto Alegre/RS. 1998.

10. Goldberg D. Genetic algorithms in search, optimization, and machine learning. [S.1.]: AddisonWesley, 1989. (Artificial Intelligence).

11. Linden R. Algoritmos Genéticos. 2a.ed. [S.1.]: Brasport, 2008.

12. Krajzewicz D, Erdmann J, Behrisch M, Bieker L. Recent Development and Applications of SUMO - Simulation of Urban MObility. International Journal On Advances in Systems and Measurements. 2012;3-4(5):128-138. 